\title{
Printed antennas: from theory to praxis, challenges and applications
}

\author{
R. Zichner ${ }^{1}$ and R. R. Baumann ${ }^{1,2}$ \\ ${ }^{1}$ Fraunhofer Institute for Electronic Nano Systems (ENAS), Technologie-Campus 3, 09126 Chemnitz, Germany \\ ${ }^{2}$ Institute for Print and Media Technology, Chemnitz University of Technology, Reichenhainer Str. 70, 09126 Chemnitz, \\ Germany
}

Correspondence to: R. Zichner (ralf.zichner@enas.fraunhofer.de) and R. R. Baumann

(reinhard.baumann@enas.fraunhofer.de, reinhard.baumann@mb.tu-chemnitz.de)

\begin{abstract}
Miniaturized, highly integrated wireless communication systems are used in many fields like logistics and mobile communications. Often multiple antenna structures are integrated in a single product. To achieve such a high level of integration the antenna structures are manufactured e.g. from flexible boards or via LDS (laser direct structuring) which allows the production of complex monopole or dipole antennas with three-dimensionally curved shapes. Main drawbacks are the sophisticated production process steps and their costs. The additive deposition of metallic inks or pastes by a printing process is an alternative manufacturing method with reduced cost.

To implement such printed antennas we investigated in the fields of antenna design, simulation, printing technology and characterization. The chosen example of use was a customized dipole antenna for a Radio Frequency Identification application. The results prove the intended functionality of the printed dipole in regard to a highly cost efficient printing manufacturing.
\end{abstract}

\section{Introduction}

Printed antennas are defined as electrically conducting structures, which are manufactured by deposition of inks or pastes onto a substrate in a single process-step. The deposition of an ink can be done by different kind of printing techniques e.g. screen printing, gravure printing or ink jet printing.

So far, printed antennas are only used in few applications. However, there is an upward trend. E.g. some applications are TV-antennas in vehicles (Toriyama et al., 1987) or radio frequency identification (RFID) transponder antennas (Zichner et al., 2010, 2011) which operate in the ultra high frequency band. In both applications the antenna structures are manufactured on curved or flexible surfaces and different materials like glass, paper or polymer foil. Standard antennas on the basis of a printed circuit board and lithographic processes are not suitable for such applications due to technical or economical reasons. Printed antennas can be manufactured flexible and on various materials/substrates. However, to provide a high performance, a dedicated design and adjustment of the antenna structures is required.

\section{Antenna design basics: from theory to praxis}

How does the design process of such adjusted antenna structures look like? Standard dipole or patch antennas can be described and dimensioned by the means of a theoretical approach and different kind of equations. According to Balanis (Balanis et al., 2005), the propagation of an E-field of an ideal, rod-shaped dipole of length $l$ can be approximated by:

$E_{\theta} \cong j \eta \frac{I_{0} e^{-j k r}}{2 \pi r}\left[\frac{\cos \left(\frac{k l}{2} \cos \theta\right)-\cos \left(\frac{k l}{2}\right)}{\sin \theta}\right]$

$\eta$ - wave impedance in free space $I_{0}$ - current $k$ - wave number: $k=2 \pi / \lambda, r-$ distance between dipole and an arbitrary point in free space $l$ - dipole length.

However, Eq. (1) is highly idealised and does not include all properties relevant to practical applications. In detail these are:

- application relevant properties:

- the antenna is applied close to dielectric materials with permittivity and permeability, which influences the wave propagation (Zichner and Baumann, 2009)

- due to specific parameters like resonance frequency, frequency bandwidth, directivity, 
impedance characteristics and antenna size the antenna design will deviate from the ideal, rod-shaped dipole

- manufacturing relevant properties:

- the used metallic inks or pastes do not offer, compared to bulk material, such high specific electrical conductivity.

Therefore, the theoretical approach of a dipole antenna does not fully comply with the practical conditions. To realize an adjusted printed antenna the knowledge in the fields of antenna design and printing processes has to be combined. The results are geometrically complex antenna designs, whose numerical description is only accomplished with enormous effort. To verify the properties of a newly designed antenna prior to manufacturing, CAD-based high frequency software tools employing finite element method and/or method of moments can be used. Besides other software tools three comprehensive tools for the calculation of the electromagnetic field propagation exist. These are "CST Studio Suite ${ }^{\mathrm{TM}}$ " (www.cst.com), "Ansys HFSS" (www.ansoft.com) and "FEKO" (www.feko.info). The first one was used for the research described hereafter.

\section{Antenna design challenges and results}

The following example of an UHF-RFID-transponder antenna shall give an insight into the challenges of the implementation of printed antennas. A printable, planar dipole antenna (see Fig. 1) was designed, based on these given parameters:

- resonance frequency: $898 \mathrm{MHz}$ (medium frequency between UHF-RFID Europe at $868 \mathrm{MHz}$ and

\section{UHF RFID Asia and USA at $928 \mathrm{MHz}$ )}

$-3 \mathrm{~dB}$ frequency bandwidth: $>=60 \mathrm{MHz}$

- radiation characteristics: omnidirectional

- antenna wave impedance: $50 \mathrm{Ohm}$

- specific properties of manufacturing process:

- printing substrate: paper, $\varepsilon_{r}=2.73, \mu_{r}=1,130 \times$ $42 \times 0.175 \mathrm{~mm}^{3}$

- expected properties of the printed conducting layer (silver, conductivity $\approx 4 \times 10^{6} \mathrm{~S} \mathrm{~m}^{-1}$, layer thickness: $4.5 \mu \mathrm{m})$

- printed antenna is applied to a $200 \times 200 \times 5 \mathrm{~mm}^{3}$ glass pane $\left(\varepsilon_{r}=4.83, \mu_{r}=1\right) @ 898 \mathrm{MHz}$.

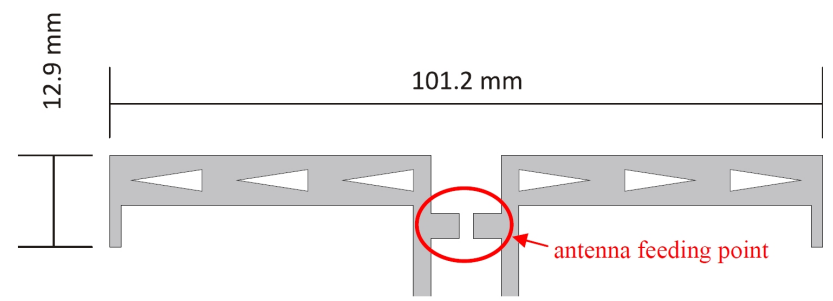

Fig. 1. Customized UHF-RFID-transponder dipole antenna design.

The main challenge was the consideration of the close distance of the antenna structure to the dielectric glass and paper materials and the implementation of the manufacturingrelevant properties which strongly influence the performance. The reason is the strong electromagnetic interaction between the antenna and the dielectric materials. Another challenge was the software-based verification of the design. Software tools are required to calculate the parameters of a complex antenna structure depending on application and manufacturing. A 3-D-model of the design including the surrounding dielectric materials was built in "CST Studio Suite $^{\mathrm{TM}}$ - Microwave Studio" (see Fig. 2). The theoretical characterization of the scenario within the tool was challenging, as the homogenous and ideal material properties used for the simulation deviate from reality and therefore were a source of error.

In addition to the designed antenna an additional circular polarized RFID-reader-patch-antenna (ANTU Patch 63) was implemented in the simulation tool to increase the compliance between theory and practice (see Fig. 3). Hence, the connection and quality of a communication link could be investigated besides the mere antenna properties. The calculated scattering parameters (both on a $50 \Omega$ port) and radiation characteristics are shown in Figs. 3 and 5.

\section{Antenna printing challenges and results}

Subsequent to the software verification of the design of the dipole UHF-RFID-transponder-antenna, the antenna is printed. The main challenge is to adjust the parameters (e.g. viscosity of ink, drying conditions, etc.) of the chosen printing process and the used conductive ink or paste to achieve a homogenous printed impression and conductive layer. Depending on the application various printing methods like inkjet, gravure printing or screen printing are possible. To achieve the defined requirements of the printed layer (silver, conductivity $\approx 4 \times 10^{6} \mathrm{~S} \mathrm{~m}^{-1}$, layer thickness: $4.5 \mu \mathrm{m}$ ) in the given example, a screen printing process was used (see Fig. 6). The applied base material was a silver paste "5064 silver conductor" by DuPont. In screen printing a printing paste is pressed through the gaps of a mesh onto the substrate underneath. The properties of the printing machine, the mesh, the substrate and the following finishing treatment 


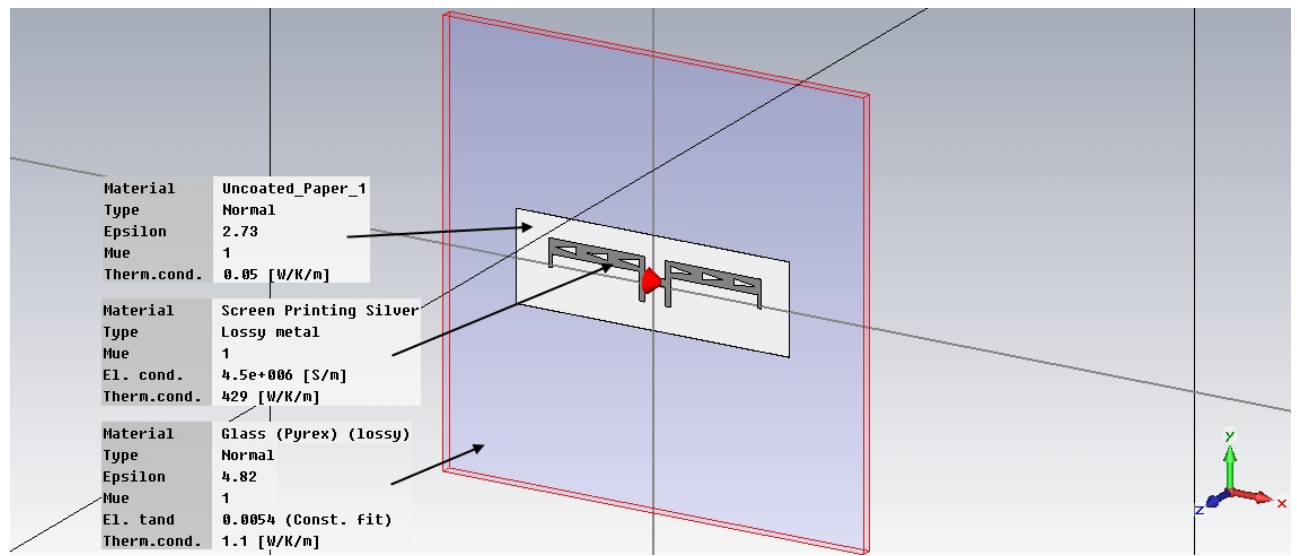

Fig. 2. UHF-RFID-transponder dipole antenna-construction (CST Microwave Studio).

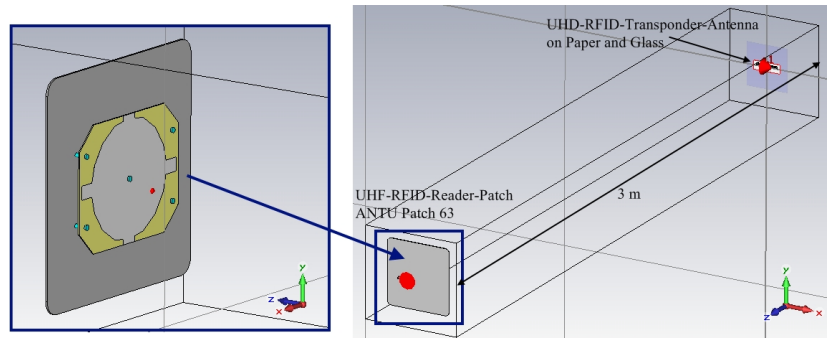

Fig. 3. Construction of a communication link between an UHFRFID-reader-patch antenna and an UHF-RFID-transponder antenna on paper attached to glass in a distance of $3 \mathrm{~m}$ (CST Microwave Studio).

have to be adjusted to the used paste. The sought results are an exact printed impression of the print master and to achieve a defined electrical conductivity.

For the manufacturing a half-automatic screen printing machine EKRA E2XL was used. The screen mesh (PET $1500 / 0^{\circ}$ tensioning bracket 77-55 YPW [77 threads per $\mathrm{cm}$, $55 \mu \mathrm{m}$ thread diameter, mesh size $67 \mu \mathrm{m}])$ was chosen according to the properties of the silver paste (viscosity: 10 $20 \mathrm{~Pa}$ s, particle size: $<2 \mu \mathrm{m}$ ). The used substrate was paper $\left(175 \mathrm{~g} \mathrm{~m}^{-2}\right)$, uncoated on both sides. Subsequent to the printing process (printing speed $0.1 \mathrm{~m} \mathrm{~s}^{-1}$ ) the layer was dried in a convection oven (Carbolite 200 by Carbolite $\mathrm{GmbH}$ ) at $120^{\circ} \mathrm{C}$ for $30 \mathrm{~min}$. After the solvents were removed from the paste the result was a dry and electrically conducting layer. The properties of the layer were determined using a tactile profilometer "Profilometer Dektak 150" by Veeco, a 4-pointmeasurement setup based on Van de Pauw consisting of a "Süss Messprober PM5" and a "Dual Channel Source Meter Keithley 2612". The measurements of the printed silver layer delivered the following properties:

- average layer thickness: $3.8 \mu \mathrm{m}$

- mean roughness Ra: $0.7 \mu \mathrm{m}$
Scattering-Parameter

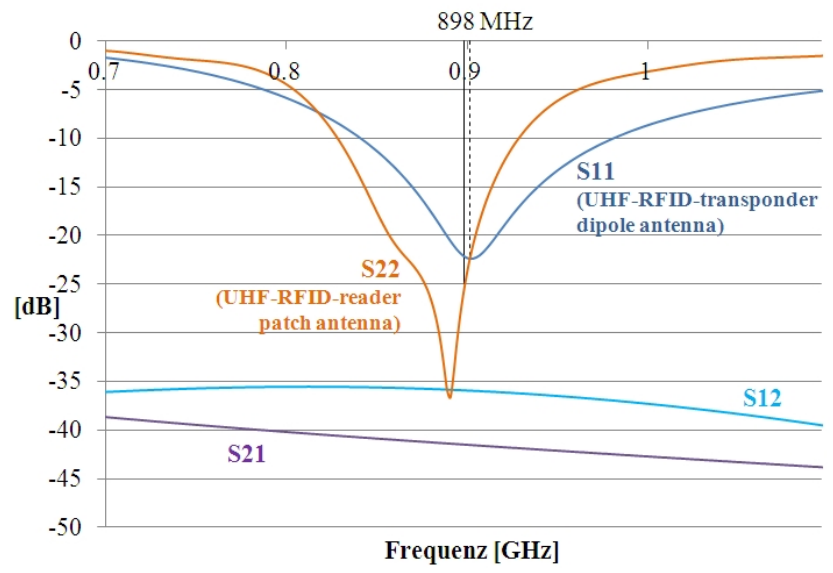

Fig. 4. Simulation results (scattering parameters) of a communication link.

- surface resistivity: $75 \mathrm{~m} \Omega / \square$

- specific electrical conductivity: $3.4 \times 10^{6} \mathrm{~S} \mathrm{~m}^{-1}$.

The printed antenna is shown in Fig. 11. As described in Sect. 3 a printed silver layer does not offer ideal homogenous properties. As a result of the use of a mesh the edges of the antenna exhibit a curved structure (see Fig. 7). However, the influence of the shape of the edges on the functionality of the dipole antenna is small, as the structures are only in a range of a few $\mu \mathrm{m}$. They are not "visible" for an incoming wave of a wave length of $33.4 \mathrm{~cm}$. This approach was investigated by a simulation. The structure shown in Fig. 7 was designed in the simulation tool (see Fig. 8) and the antenna properties were calculated. The simulation scenario included the design of the UHF-RFID-transponder-antenna, the substrate and the dielectric glass material. 


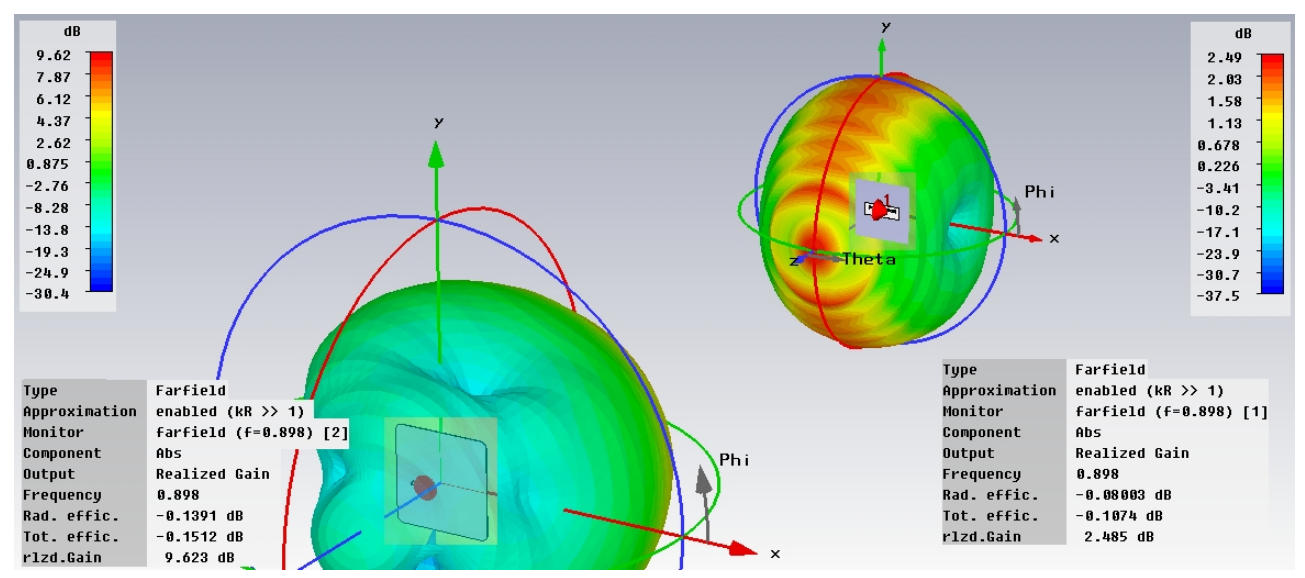

Fig. 5. Simulation results (radiation characteristics, realized gain) of a communication link.

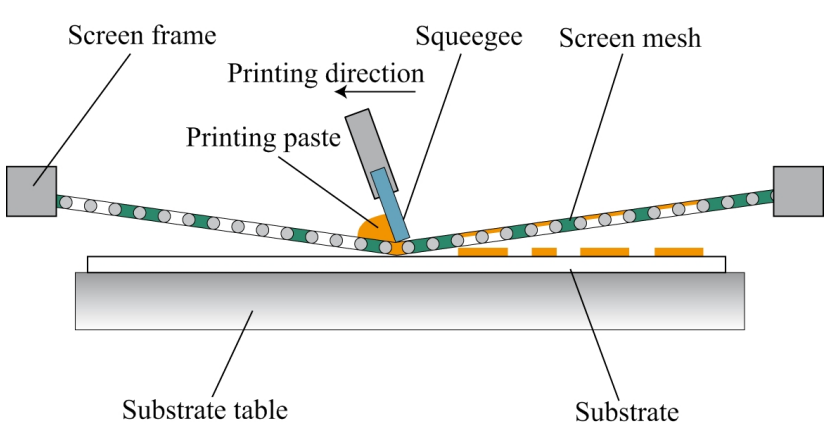

Fig. 6. Schematic plot of screen printing.

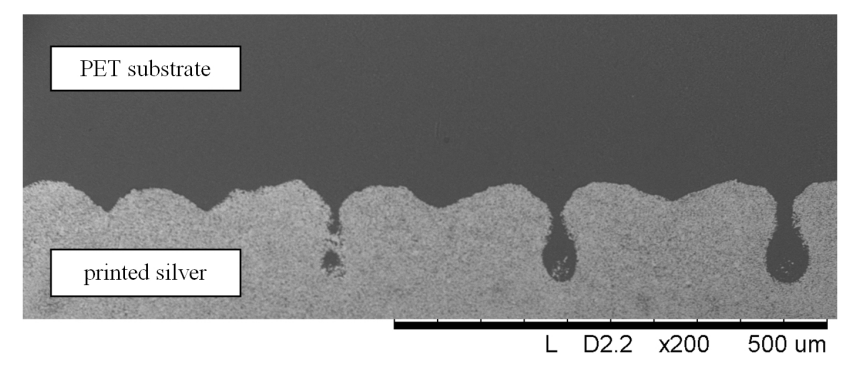

Fig. 7. SEM picture of a printed silver antenna edge on a PET substrate.

The S11-parameter (see Fig. 9) shows only a little displacement of the resonance frequency by $3.6 \mathrm{MHz}$. The reason is the reduction of the antenna area by $-0.6 \%$. The loss of $3.1 \mathrm{~dB}$ of the scattering parameters results from the surface currents at the edge structures (see Fig. 10).

\section{Antenna measurement challenges and results}

Subsequent to the manufacturing of the dipole antenna on a paper substrate, it was applied onto a glass pane and analyzed in an anechoic chamber with a network analyzer "ZVL6" by

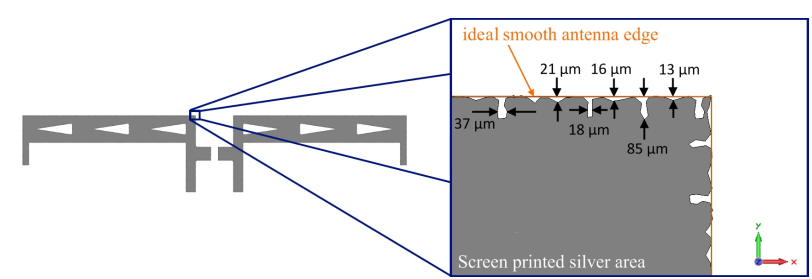

Fig. 8. Reconstruction of screen printing edge behavior in the simulation tool.

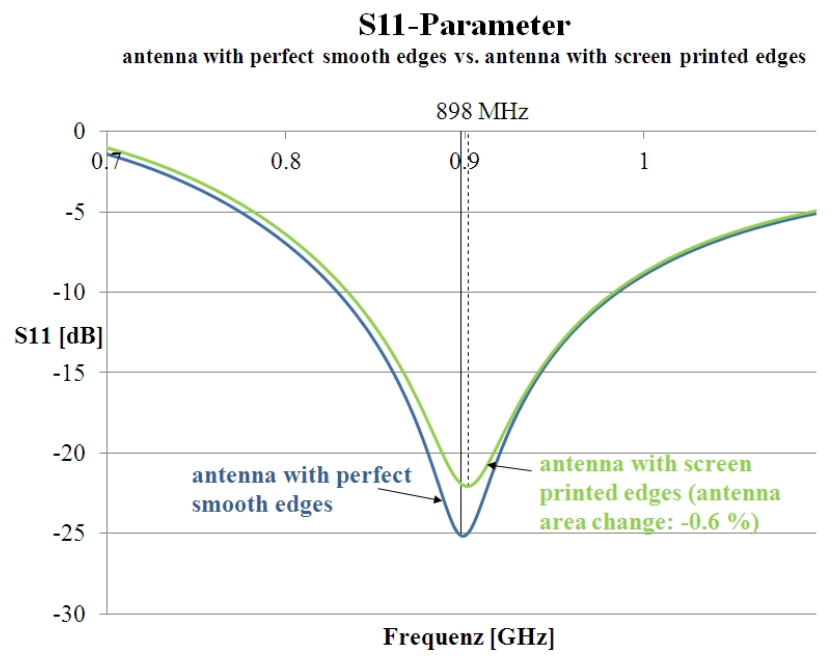

Fig. 9. Simulation results: S11-parameter of printed antennas with perfect smooth (blue) vs. screen printed (green) edges.

Rohde \& Schwarz. The antenna was connected at the feeding point by a coax cable (see Fig. 11). The electrical contact of a printed silver layer is difficult as a soldering is not possible. To reliably ensure the electrical contact an anisotropic, electrical conducting tape (3M Electrically Conductive Adhesive Transfer Tape \#9703 8017 086) was used. 


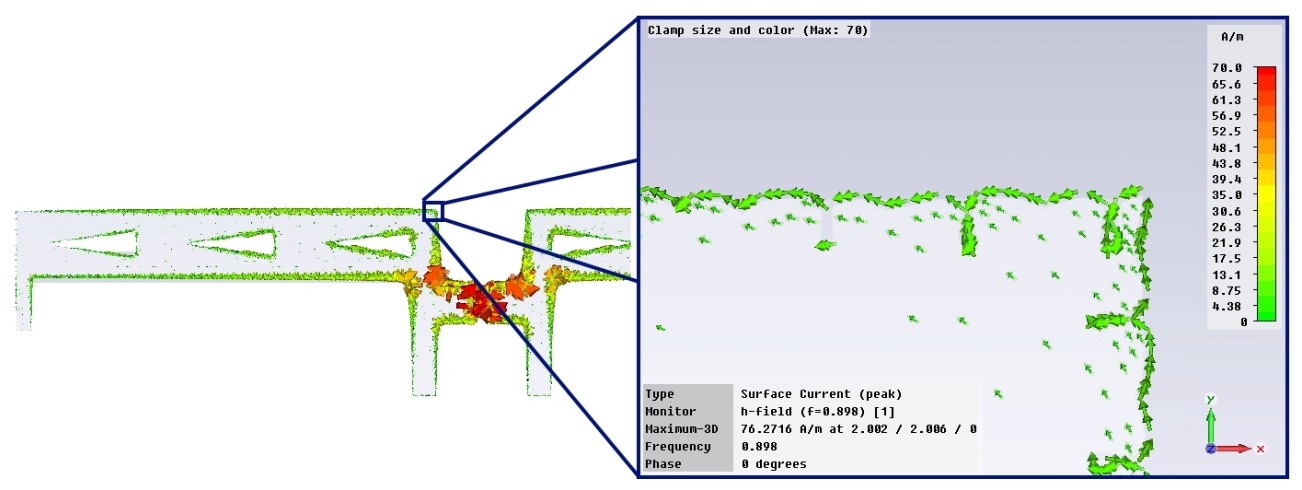

Fig. 10. Simulation results: surface current behavior in dependency of the screen printed edges.

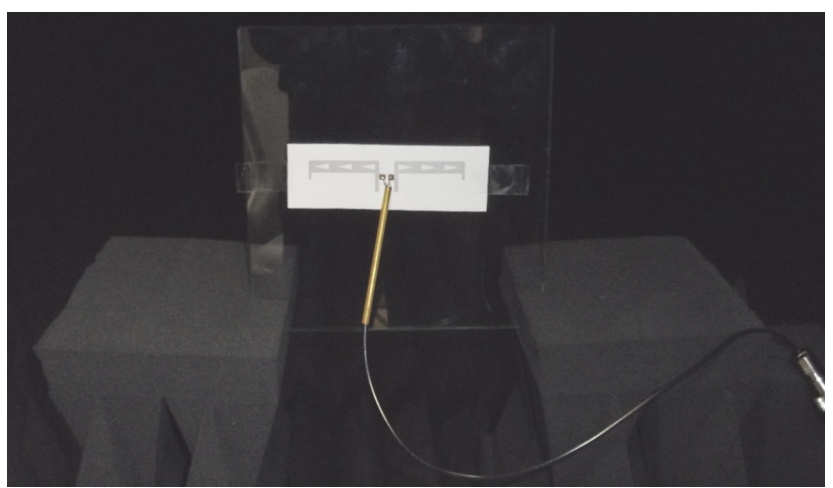

Fig. 11. Measurement setup of a printed antenna on paper attached to glass in an anechoic chamber.

\section{S11 Paramter: Simulation vs. Measurement}

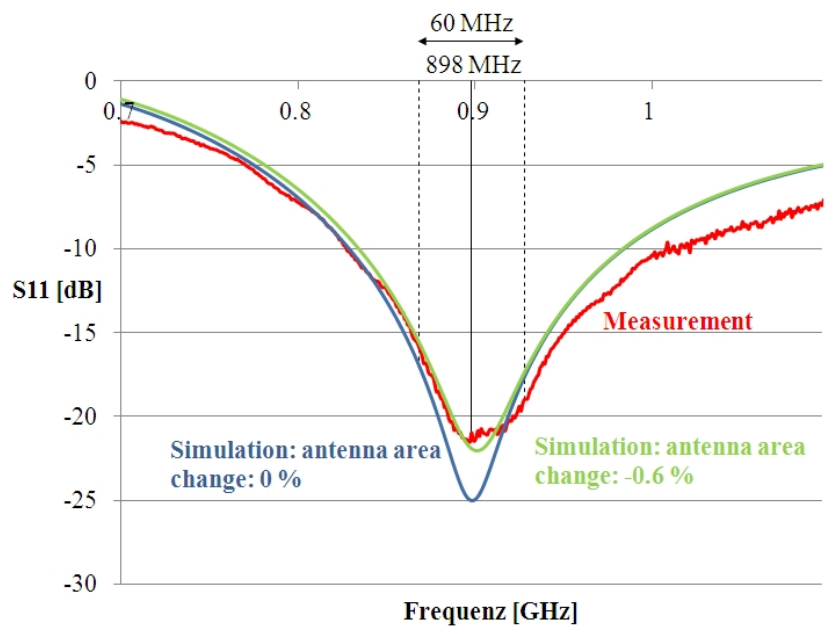

Fig. 12. S11 parameter: simulation vs. measurement.

The measurement results of the network analyzer and the simulation results of the scattering behavior are given in Fig. 12. The measurement results are close to the simulation results of the antenna with a reduced area of $-0.6 \%$ (due to

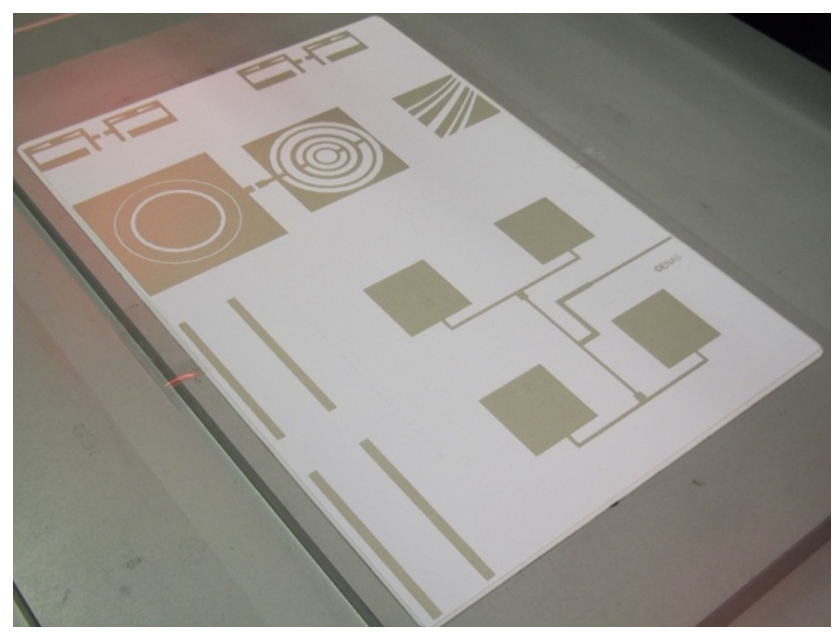

Fig. 13. Printed antennas on a flexible paper substrate.

the edges from the screen printing). The measurements confirm the simulations.

\section{Conclusions}

The paper demonstrates the high potential of a printed dipole antenna structure. Challenges like antenna design adaption to dielectric environments and printed layer parameters are shown. Furthermore, we investigated a complex dipole antenna design, which takes into account the application defined parameters like the resonance frequency of $898 \mathrm{MHz}$, the permittivity and permeability of the printing substrate as well as the electrical parameters of the printed layer. After the design process we constructed a fully parameterized 3D-model to simulate the antenna characteristics in respect to the application scenario. The consecutive screen printing process offers an antenna manufacturing process that effects the antenna edge shapes in a way that the effective antenna area is reduced compared with an etching process. This fact 
influences the scattering parameters, what was verified in the simulations and measurements.

In summary, it can be stated that utilizing printing technology, highly sophisticated antennas can be manufactured in a low cost production process.

\section{References}

Balanis, C. A.: Atenna Theory, Third Edition, JohnWiley \& Sons, 162-184, ISBN: 0-471-66782-X, 2005.

Toriyama, H., Ohe, J., Kondo, H., and Yotsuya, H.: Development of Printed-On Glass TV Antenna System for Car, IEEE, 334-342, 1987.
Zichner, R. and Baumann, R. R.: Customised Printed RFID Antennas for Various Environments, Proc. LOPE-C, p. 3.11, 2009.

Zichner, R., Siegel, F., Hösel, M., and Baumann, R. R.: Optimized antenna design for gravure printed RFID Applications, Proc. LOPE-C, 13-16, 2010.

Zichner, R., Siegel, F., Hösel, M., and Baumann, R. R.: Communication Quality of printed UHF RFID Transponder Antennas, Proc. LOPE-C, 361-363, 2011. 